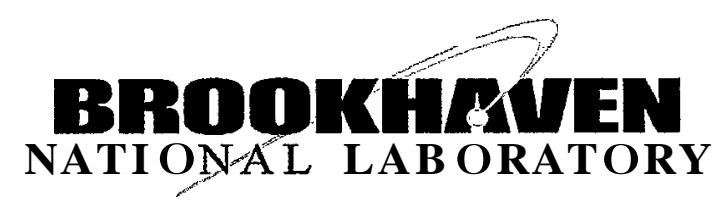

BNL-73479-2005-CP

\title{
Interaction Region Designfor the Electron - Light Ion Collider ELIC
}

\author{
C. Montag, et al \\ Presenfed at fhe Particle Accelerafor Conference(PAC'05) \\ Knoxville, Tennessee \\ May 16-20,2005
}

\author{
Collider-Accelerator Department \\ Brookhaven National Laboratory \\ P.O. Box 5000 \\ Upton, NY 11973-5000 \\ www.bnl.gov \\ Managed by \\ Brookhaven Science Associates, LLC \\ for the United States Department of Energy under \\ Contract No. DE-AC02-98CH10886
}

This is a preprint of a paper intended for publication in a journal or proceedings. Since changes may be made before publication, this preprint is made available with the understanding that it will not be cited or reproduced without the permission of the author. 


\section{DISCLAIMER}

This report was prepared as an account of work sponsored by an agency of the United States Government. Neither the United States Government nor any agency thereof, nor any of their employees, nor any of their contractors, subcontractors, or their employees, makes any warranty, express or implied, or assumes any legal liability or responsibility for the accuracy, completeness, or any third party's use or the results of such use of any information, apparatus, product, or process disclosed, or represents that its use would not infringe privately owned rights. Reference herein to any specific commercial product, process, or service by trade name, trademark, manufacturer, or otherwise, does not necessarily constitute or imply its endorsement, recommendation, or favoring by the United States Government or any agency thereof or its contractors or subcontractors. The views and opinions of authors expressed herein do not necessarily state or reflect those of the United States Government or any agency thereof.

FOR UNCLASSIFIED, UNLIMITED STIPRODUCTS

Available electronically at:

OSTI:

\section{http://www.osti.gov/bridge}

Available for a processing fee to U.S. Department of Energy and its contractors, in paper from:

U.S. Department of Energy

Office of Scientific and Technical Information

P.O. Box 62

Oak Ridge, TN 37831

Phone: (865) 576-8401

Facsimile: (865) 576-5728

E-mail: reports@adonis.osti.gov

National Technical Information Service(NTIS):

Available for sale to the public from:

U.S. Department of Commerce

National Technical Information Service

5285 Port Royal Road

Springfield, VA 22131

Phone: (800) 553-6847

Facsimile: (703) 605-6900

Online ordering: http://www.ntis.gov/ordering.htm 


\title{
INTERACTION REGION DESIGN FOR THE ELECTRON -LIGHT ION COLLIDER ELIC *
}

\author{
C. Montag, Brookhaven National Laboratory, Upton, NY 11973, USA \\ A. Bogacz, Y. Derbenev, L Merminga, ЛLab, Newport News, USA
}

\begin{abstract}
The Electron-LightIon Collider ELIC proposed by Jefferson Lab aims at a luminosity in the $10^{35} \mathrm{~cm}^{-2} \mathrm{sec}^{-1}$ range for collisions of $150 \mathrm{GeV}$ protons on $7 \mathrm{GeV}$ electrons $[1,2]$. To achieve these high luminosities, very strong low- $\beta$ focusing of low-emittance beams is required. Taking advantage of the unequal design proton beam emittances in the two transverse planes, an interaction region design based on superconducting quadrupole doublets has been developed. Compared with the original design in [2], this scheme provides larger beam apertures at lower magnetic fields, while potentially doubling the luminosity.
\end{abstract}

\section{INTRODUCTION}

Jefferson Lab has proposed the CEBAF-based ElectronLight Ion Collider ELIC to provide the worldwide nuclear physics community with a high-luminosityelectronion collider with center-of-mass energies from $20 \mathrm{GeV}$ to $65 \mathrm{GeV}$. This machine consists of a figure- 8 shaped $7 \mathrm{GeV}$ electron circulator ring, and a similarly shaped $150 \mathrm{GeV}$ polarized ion storage ring. Four interaction regions are located in the straight sections. Low $\beta$ focussing to $\beta$ functions on the order of $\beta^{*}=5 \mathrm{~mm}$ at the interaction points (IP) and bunch collision rates of $1.5 \mathrm{GHz}$ are required to achieve luminosities of $10^{35} \mathrm{~cm}^{-2} \mathrm{sec}^{-1}$. Some basic parameters of ELIC according to Ref. [2] are listed in Table 1.

Since a bunch spacing of only $20 \mathrm{~cm}$ is planned, a large crossing angle of $100 \mathrm{mrad}$ is required to avoid parasitic collisions. Without separation dipoles that would generate intense synchrotronradiation, the interactionregion design is greatly simplified. The use of commonmagnets for both beams is also unnecessary because the separation between the two beams is large enough to guide either one of the beams through a field-freeregion of the focussing elements for the other beam even at the innermost quadrupoles.

\section{DESIGN CONSIDERATIONSAND OPTICS}

A distinctivedesign feature of ELIC is the use of unequal hadron beam emittances in the two transverse planes, as it results naturally from intra-beam scattering and electron cooling in a flat, (locally) decoupled storage ring.

When these beams are focussed to the same $\beta$-functions in both planes at the $\operatorname{IP}, \beta_{x}^{*}=\beta_{y}^{*}$, the resulting beambeam tuneshift in the vertical plane of both beams becomes

\footnotetext{
${ }^{*}$ Work performed underthe auspices of the U.S. Department of Energy
}

\begin{tabular}{lcc}
\hline \hline parameter & EPAC [2] & "NEW" \\
\hline energy E $(\mathrm{p} / \mathrm{e})[\mathrm{GeV}]$ & $150 / 7$ & $\mathbf{1 5 0 / 7}$ \\
\# part.//unch $\mathrm{N}(\mathrm{p} / \mathrm{e})\left[10^{10}\right]$ & $\mathbf{0 . 4 / 1 . 0}$ & $\mathbf{0 . 4 / 1 . 0}$ \\
collision rate [GHz] & 1.5 & 1.5 \\
emittance $\mathrm{E}(\mathrm{x} / \mathrm{y})[\mathrm{nm}]$ & 6.310 .25 & 6.310 .25 \\
$\beta^{*}(\mathrm{x} / \mathrm{y})[\mathrm{mm}]$ & $\mathbf{5 / 5}$ & 2515 \\
$\sigma^{*}(\mathrm{x} / \mathrm{y})[\mu \mathrm{m}]$ & 5.811 .2 & 13.011 .2 \\
beam-beam tuneshift $\xi_{h}(\mathrm{x} / \mathrm{y})$ & $0.002 / 0.01$ & $\mathbf{0 . 0 0 2 / 0 . 0 0 5}$ \\
beam-beam tuneshift $\xi_{\mathrm{e}}(\mathrm{x} / \mathrm{y})$ & $\mathbf{0 . 0 1 7 / 0 . 0 8 6}$ & $\mathbf{0 . 0 1 9 / 0 . 0 4 3}$ \\
\hline lumin. $\mathcal{L}\left[10^{34} \mathrm{~cm}^{-2} \mathrm{sec}^{-1}\right]$ & 7.7 & 3.4 \\
\hline
\end{tabular}

Table 1: Parameters of the Electron-Light Ion Collider ELIC. The column labeled 'EPAC' shows the design parameters presented in Ref.[2], while the parameters used for the design presented in this paper are listed in the column labeled "NEW". Luminosities are given for equal bunch intensities in both designs. However, in the "NEW" design, bunch currents can potentially be doubled due to the smallerbeam-beam tuneshift resulting from unequal $\beta$ functions in the two planes.

much larger than in the horizontal plane, $\xi_{y} \gg \xi_{x}$. Without single-bunch instabilities the maximumbunch intensity is therefore limited by the larger beam-beam tuneshift parameter \& , regardless of the actual of \&. A more balanced situation with nearly equal beam-beam tuneshift parameters in the two planes is therefore more desirable.

With the beam-beam tuneshift parameter given as

$$
\xi_{x, y}=\frac{N \dot{r_{c}} \beta_{x, y}^{*}}{2 \pi \gamma \sigma_{x, y}\left(\sigma_{x}+\sigma_{y}\right)},
$$

where $N, r_{c}$, and $\gamma$ denote the number of particles in the oncoming beam, the classical particle radius and the Lorentz factor, respectively, this can be accomplished by increasingthe horizontal $\beta$-function $\beta_{x}^{*}$. This must be done in both electron and hadron rings in order to keep equal beam sizes at the IP. The resulting increase of the horizontal beam size $\sigma_{x}^{*}$ of the oncoming beam reduces the vertical beam-beam tuneshift parameter $\xi_{y}$, while the horizontal tuneshift remains practically constant as long as $\sigma_{x}^{*} \gg \sigma_{y}^{*}$.

With $\beta$-functions focused down to $\beta_{x}^{*}=25 \mathrm{~mm}$ and $\beta_{y}^{*}=5 \mathrm{~mm}$ at the $\mathrm{P}$ by means of quadrupole doublets, beam-beam tuneshift parameters of $\xi_{x}=0.019$ and $\xi_{y}=$ $\mathbf{0 . 0 4 3}$ are achieved in the design presented here (See Table 1). The higher energy hadron beam is focused by a superconductingquadrupole doublet located $2 \mathrm{~m}$ from the IP. The first quadrupole of this doublet, QHI, focuses in 



Figure 1: Hadron (top) and electron (bottom) interaction region lattices. The electron low- $\beta$ doublet fits in the gap between the two quadrupoles of the hadron low- $\beta$ doublet, minimizing the required transverse separation of the two beam lines. The third magnet shown in the electron lattice is used to match this interactionregion to the regular optics of the arc.

the vertical plane where the $\dot{\beta}$-function rises more rapidly due to the smaller $\beta$-function at the IP, see Figure 1 . The length of this magnetneeds to be minimized to avoid aperture problems due to the rapidly growing, defocused horizontal $\beta$-function. At a length of $l=1.2 \mathrm{~m}$, a strength of $k=-0.5 \mathrm{~m}^{-2}$, and a maximum horizontal $\beta$-function of $\hat{\beta}_{x}=677 \mathrm{~m}$, a minimum aperture of $12 \sigma_{h}$ can be achieved with a peak field of 6.2T.

The second lens, $\mathrm{QH} 2$, focuses horizontally, with a maximum horizontal $\beta$-function inside the lens of $\hat{\beta}_{x}=$ $5750 \mathrm{~m}$. With a length of $2=3.0 \mathrm{~m}$ and a strength of $k=0.12 \mathrm{~m}^{-2}$, this magnet has a peak field of $4.3 \mathrm{~T}$ for $12 \sigma_{h}$ minimum aperture. Table 2 lists the parameters of the hadron doublet.

The drift between the two hadron quadrupoles provides sufficient space for the electron low- $\beta$ doublet, as depicted in Figure 1. This arrangement minimizes the required crossing angle by avoiding cryostats for both beams be-

\begin{tabular}{lcc}
\hline \hline parameter & QH1 & QH2 \\
\hline length $l[\mathrm{~m}]$ & $\mathbf{1 . 2}$ & $\mathbf{3 . 0}$ \\
strength $k\left[\mathrm{~m}^{-2}\right]$ & -0.5 & $\mathbf{0 . 1 2}$ \\
distancefrom $\mathrm{IP} s[\mathrm{~m}]$ & $\mathbf{2 . 0}$ & $\mathbf{5 . 0}$ \\
a $[\mathrm{m}]$ & $\mathbf{6 7 7}$ & $\mathbf{5 7 0 0}$ \\
peak field $\boldsymbol{B}[\mathrm{T}]$ & $\mathbf{6 . 2}$ & $\mathbf{4 . 3}$ \\
quad type & $\mathrm{d}$ & $\mathrm{f}$ \\
minimum aperture $\left[\sigma_{h}\right]$ & $\mathbf{1 2}$ & $\mathbf{1 2}$ \\
\hline
\end{tabular}

Table 2: Hadron low- $\beta$ quadrupole doublet parameters.

\begin{tabular}{lcc}
\hline \hline parameter & QE1 & QE2 \\
\hline length $l[\mathrm{~m}]$ & $\mathbf{0 . 6}$ & $\mathbf{0 . 7}$ \\
strength $k\left[\mathrm{~m}^{-2}\right]$ & $\mathbf{- 1 . 4}$ & $\mathbf{1 . 1}$ \\
distance from IP s $[\mathrm{m}]$ & $\mathbf{3 . 3}$ & 4.2 \\
$\hat{\beta}_{x}[\mathrm{~m}]$ & $\mathbf{9 2 9}$ & $\mathbf{2 1 5 3}$ \\
peak field $\boldsymbol{B}[\mathrm{T}]$ & $\mathbf{1 . 6}$ & $\mathbf{1 . 9}$ \\
quad type & $\mathrm{d}$ & $\mathrm{f}$ \\
minimum aperture $\left[\sigma_{h}\right]$ & 20 & $\mathbf{2 0}$ \\
\hline
\end{tabular}

Table 3: Parameters of the electron low- $\beta$ quadrupole doublet.

ing side-by-side at the same longitudinal position. The first, vertically focussing magnet of this doublet (QE1) is $l=0.6 \mathrm{~m}$ long and has a strength of $k=1.4 \mathrm{~m}^{-2}$. With the maximum horizontal $\beta$ function inside that magnet being $\hat{\beta}_{x}=929 \mathrm{~m}$, the peak field of this magnet is $1.6 \mathrm{~T}$ for a minimumaperture of $20 \sigma_{e}$.

The second, horizontally focussing magnet of the electron doublet, QE2, is $l=0.7 \mathrm{~m}$ long and has a strength of $k=1.1 \mathrm{~m}^{-2}$. Together with a maximum horizontal $\beta$ function of $\hat{\beta}_{x}=2150 \mathrm{~m}$, a minimum aperture of $20 \sigma_{e}$ can be accomplishedby a peak field of $1.9 \mathrm{~T}$. The parameters of the electron low- $\beta$ doublet are listed in Table 3 .

\section{SYNCHROTRONRADIATION}

Since there are no dipoles in the detector vicinity, synchrotron radiation in this area is only generated in quadrupoles. For well-steered beam, the core of the beam where the majority of the electrons is located experiences only relatively low magnetic fields and therefore generates only soft photons. For example, electrons having a la, amplitude experience magnetic fields of $0.1 \mathrm{~T}$, which produces synchrotronradiation photons with a critical energy of $E,=3.3 \mathrm{keV}$. A small number of electrons at an amplitude of $20 \sigma_{e}$ in the transverse tails, however, undergoes focussing by magnetic fields up to some $2 \mathbf{T}$, thus generating $E$, $=65 \mathrm{keV}$ photons.

Since the total synchrotronradiationpower generatedby electrons in the transverse tails is quite small due to the small fraction of electrons at large amplitudes, this portion of the synchrotron radiation fan can be easily collimated upstream of the detector, protecting the detector from high- 
energy photons. These collimators will be placed where the horizontal beam size is small while the fan is wide, ensuring sufficient free aperture for:the beam.

Synchrotronradiation photons generated by the core of the beam stay mostly within the 200 , electron beam itself and therefore do not hit any detector components. This is illustrated in Figure 2, which depicts the 200, electron beam envelopes and the corresponding synchrotron radiation fans generated by the $5 \sigma_{e}$ electron beam core, in both the horizontal and vertical planes.

In the vertical plane, the fan generated upstream of the IP can be collimated at the entrance of the central detector, at $s=-2.0 \mathrm{~m}$, because at this location the 200 , electron beam is narrower than the fan from the $5 \sigma_{e}$ beam. The innermost quadrupole QE1 generates a fan that is somewhat outside the $20 \sigma_{e}$ beam on the outgoing side at distances beyond about $4 \mathrm{~m}$, but still well within the aperture of the correspondingmagnet.

In the horizontal plane, however, the situation is somewhat more difficult. The strong focusing of the second quadrupole, QE2, generates a synchrotron radiation fan with a "waist" around $s=-2 \mathrm{~m}$. This fan becomes very wide on the outgoing side and does not easily fit into the quadrupole magnet apertures there. Possible remedies are larger magnet apertures, which are feasible due to the relatively low gradient of these magnets, and/or installation of a synchrotron radiation collimator at $s=-2 \mathrm{~m}$ that also reduces the free aperture for the electron beam to about $10 \sigma_{e}$. This aperture reduction is not expected to have any negative effects because the electron beam is circulating for a few hundred turns at most. To avoid additional detector background from electrons scattering off this collimator, an additional beam collimation system away from the IPs is advisable that defines the overall beam aperture in the machine.

\section{CONCLUSION}

An alternativeinteraction region design for the ElectronLight Ion Collider ELIC has been presented. Using unequal @-functionsin the two transverse planes at the interaction point, this scheme provides about half the luminosity of the previous design outlined in [2], given the same bunch intensities. The magnetic fields of the superconducting low- $\beta$ quadrupoles for the hadron beam are significantly lower than in the previous design, while simultaneously doubling the aperture in terms of rms beam size.

Additionally, this scheme leads to beam-beam parameters that are roughly a factor two below those of the previous design. Assuming that this is the only limitation, this allows to double the bunch intensities of both the electron and the hadron beam, thus approximatelydoubling the luminosity of the previous design to $13.6 .10^{34} \mathrm{~cm}^{-2} \mathrm{sec}^{-1}$.

Doubling the bunch intensities also allows for doubling the $\beta$-functions at the $\mathbb{P}$, thus reducing the field strengths of the low- $\beta$ quadrupoles while providing the same luminosity as in the previous design.
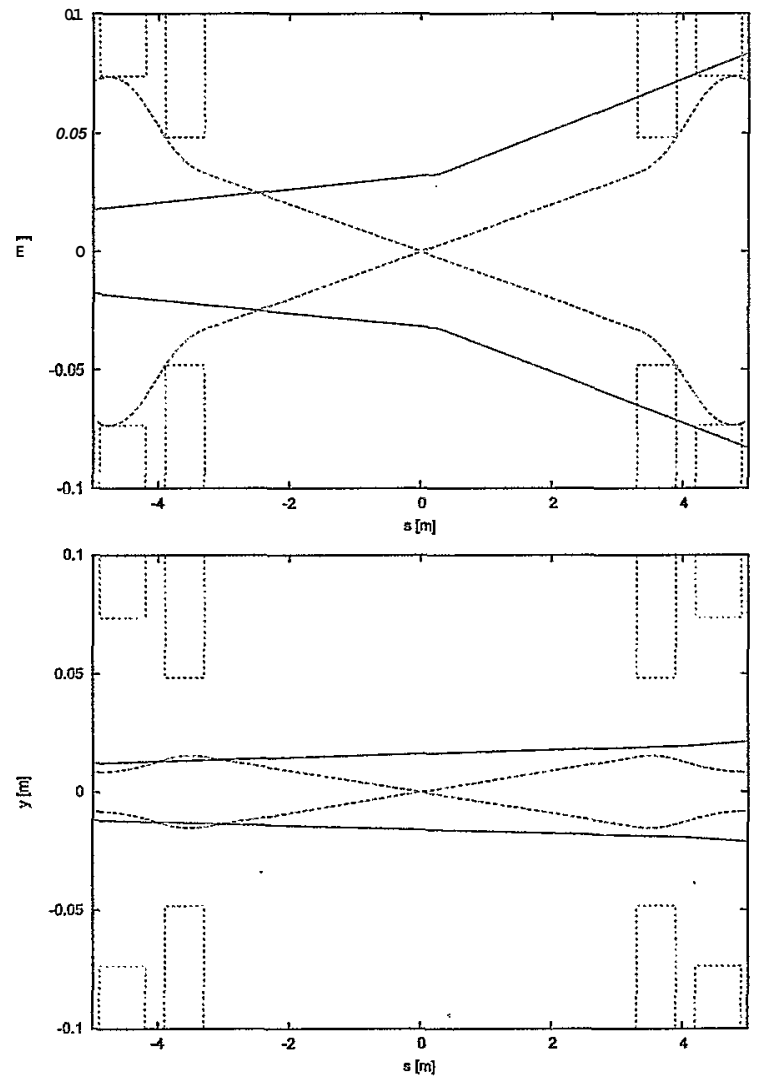

Figure 2: 200 electron beam envelopes (green) and synchrotronradiation fans as generated by particles at 50 (red). The superconductinglow- $\beta$ magnets are indicated in blue. The electron beam direction is from left to right. Upper plot - horizontal plane, lower plot - vertical plane.

\section{REFERENCES}

[1] Ya. Derbenev, ICFA Beam Dynamics Newsletter, No. 30, 2003

[2] Ya. Derbenev et al., "Electron-IonCollider at CEBAF: New Insights and Conceptual Progress", Proc. EPAC 2004 